\title{
Enterovirus D68 Subclade B3 in Children with Acute Flaccid Paralysis in West Africa, 2016
}

Amary Fall, Ndack Ndiaye, Kevin Messacar, Ousmane Kebe, Mamadou Malado Jallow, Hamid Harouna, Davy Evrard Kiori, Sara Sy, Déborah Goudiaby, Mohamed Dia, Mbayame Ndiaye Niang, Kader Ndiaye, Ndongo Dia

We tested for enterovirus D68 in fecal samples collected during June-September 2016 from 567 patients with acute flaccid paralysis in 7 West Africa nations. Children $<5$ years old comprised $64.3 \%$ of enterovirus D68 positive patients. Our findings emphasize the need for active surveillance for acute flaccid myelitis.

Uis Tntil 2014, enterovirus D68 (EV-D68) infections had been identified only sporadically after its discovery in 1962, but since 2014, the virus has emerged to cause large outbreaks of respiratory disease worldwide. In recent years, EV-D68 has been reported in outbreaks in the United States, Canada, Europe, Asia, and Africa, affecting $\geq 2,287$ persons worldwide (1-4).

The 2014 EV-D68 outbreak coincided temporally and geographically with increases in cases of acute flaccid myelitis (AFM), a subtype of acute flaccid paralysis (AFP), described by the Centers for Disease Control and Prevention as acute-onset flaccid weakness, combined with spinal cord lesions confirmed by magnetic resonance imaging, largely restricted to the gray matter, and spanning $\geq 1$ spinal segments (5). In 2014, a total of 120 AFM cases in the United States $(4,6)$ and $\geq 6$ in Europe were associated with EV-D68 outbreaks. Subsequent biennial circulation of EV-D68 was associated with surges in AFM cases in the United States in 2016 and 2018 (7). In addition, 29 EV-D68-associated AFM cases were reported in Europe in 2016 (8). Africa, unlike Europe and the United States, has no active AFM surveillance. However, a 2016 study in Senegal reported 4 cases of paralysis associated with

Author affiliations: Institute Pasteur Dakar, Senegal (A. Fall, N. Ndiaye, O. Kebe, M.M. Jallow, D.E. Kiori, S. Sy, D. Goudiaby, M. Dia, M.N. Niang, K. Ndiaye, N. Dia); University of Colorado, Aurora, Colorado, USA (K. Messacar); Ministère de la Santé Publique, Niamey, Niger (H. Harouna)

DOI: https://doi.org/10.3201/eid2609.200312
EV-D68 identified in fecal samples from children with AFP (2). With no AFM- or AFP-specific surveillance data available, we analyzed fecal samples collected for polio surveillance to better understand the extent of EV-D68 associated with AFP in West Africa and the genetic diversity of identified strains.

\section{The Study}

We retrospectively tested for EV-D68 in fecal samples from patients $<15$ years old with AFP. The samples were collected during June-September 2016 as part of routine poliomyelitis surveillance in Niger, Senegal, Guinea, Mauritania, Gambia, Guinea-Bissau, and Cape Verde. Specimens were collected 24-48 hours apart and $\leq 14$ days of paralysis onset. We inoculated fecal specimens onto human rhabdomyosarcoma cells after using chloroform for EV isolation according to the procedures described in the laboratory manual for the World Health Organization's Global Polio Laboratory Network (http://polioeradication.org/wp-content/uploads/2017/05/Polio_Lab_Manual04.pdf). We used a QIAmp Viral RNA Mini Kit (QIAGEN, https://www.qiagen.com) to extract RNA from $200 \mu \mathrm{L}$ clarified fecal suspensions pretreated with

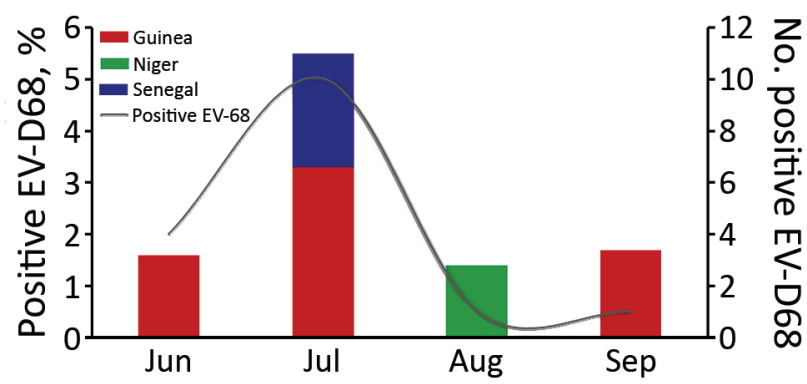

Figure 1. EV-D68 detection in fecal samples from patients with acute flaccid paralysis in 3 West Africa countries, June to September 2016. EV-D68, enterovirus D68. 


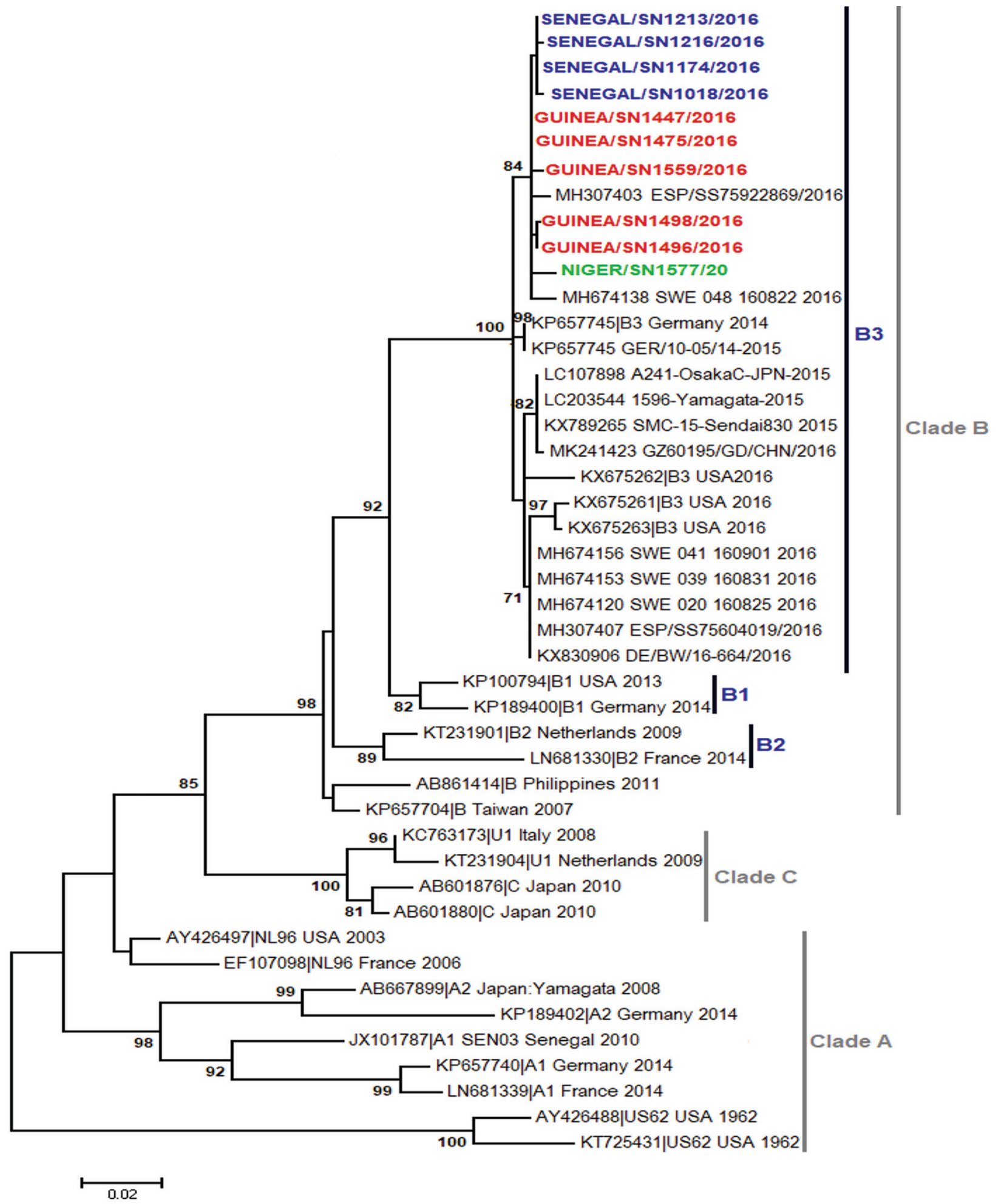

Figure 2. Phylogenetic relationships among EV-D68 strains detected in Guinea (red), Niger (green), and Senegal (blue), JuneSeptember 2016. We used the maximum-likelihood method based on the Tamura-Nei model method in MEGA7 (http://www. megasoftware.net) to generate the phylogenetic tree constructed on the viral protein 1 region of EV-D68 strains. Sequences are identified by GenBank accession number, country, and period of detection. The phylogenetic tree is rooted by the oldest EV-D68 sequence in GenBank, the Fermon strain. We performed 1,000 bootstrap replications to determine the consensus tree; support for nodes present in $>70 \%$ of the trees are annotated. EV-D68, enterovirus D68. 
chloroform. After RNA extraction, we screened all samples for EV-D68 by real time reverse transcription PCR as described elsewhere (2). For molecular characterization, the viral protein 1 region was amplified by a nested PCR and sequenced as described elsewhere (2). The alignment and phylogenic analyses of sequences obtained after cleaning were performed using MEGA 7.0 software (https://www. megasoftware.net).

We tested for EV-D68 in 567 fecal samples collected from 7 countries in West Africa: Cape Verde $(n=1)$, Gambia $(n=6)$, Guinea-Bissau $(n=5)$, Guinea Conakry $(n=391)$, Mauritania $(n=20)$, Niger $(n=$ $85)$, and Senegal $(n=59)$, during June-September 2016. EV-D68 was detected in $16(2.8 \%)$ patients from 3 countries: Guinea (11/391), Niger (1/85), and Senegal (4/59). The detection of EV-D68 in fecal samples from AFP patients in West Africa countries is consistent with case reports and case series from the United States, South America, Asia, and Europe during the same period (7-11).

The first EV-D68-associated AFP case was detected in Guinea in June 2016. Most cases (10/16) in West Africa in 2016 were detected during July (Figure 1), similar to the seasonality that has been observed in several other countries, including the United States (12), the Netherlands (13), and Senegal (3). Most EV-D68-positive patients (64.3\%) were children $<5$ years old, consistent with our previous report from Senegal $(2,3)$. BLAST analysis (https:/ / blast.ncbi.nlm.nih.gov/Blast.cgi) showed that all sequenced EV-D68 strains shared $>98 \%$ homology with strains detected in Spain, Sweden, Germany, Japan, and China. Phylogenetic analysis of the viral protein 1 fragment revealed that all sequences from West Africa belonged to clade B, subclade B3 (Figure 2). Indeed, EV-D68 subclade B3 was the predominant strain reported in several global regions during the same period (9-14). Moreover, results from phylogenetic testing showed that EV-D68 strains in West Africa clustered with strains circulating in Spain (GenBank accession no. MH307403) and Sweden (accession no. MH674138), with a bootstrap value of 97 .

Our study has some limitations. One ongoing issue is the inability to accurately describe the flaccid paralysis syndrome. Radiography imaging will probably help distinguish AFM from other AFP conditions. In addition, detecting EV-D68 in feces does not prove a causal relationship with AFM, although in this study all of the fecal samples tested negative for poliovirus and other enteroviruses, ruling out those possible alternative diagnoses.
The absence of EV-D68 positive patients from the other West Africa countries may be due to the small number of samples collected and screened during the study period. EV-D68 prevalence in West Africa might be higher if respiratory samples, known to yield higher EV-D68 counts than fecal samples, were used for screening $(6,14)$. Recently, the Pan American Health Organization and the World Health Organization provided updated recommendations to include respiratory sampling in suspected AFP cases (15).

\section{Conclusions}

This study provides evidence of more widespread EV-D68 circulation in West Africa in 2016 than previously reported. Enhanced surveillance for EV-D68, including collecting respiratory specimens from patients with confirmed cases of AFM, is needed to improve our understanding of this disease and its burden. Phylogeographic and phylodynamic studies based on full genomes are needed to better understand the introduction of EV-D68 in Africa during these different outbreaks.

\section{Acknowledgments}

We thank Mohamed Salif Sylla, Dabo Moustapha, Djenou Sompare, and Kathleen Victoir (DI-IPP) and acknowledge the US Centers for Disease Control and Prevention for its unwavering support.

This study was supported by the US Department of Health and Human Services through grant number IDSEP140020-01-00 via the International Division of Pasteur Institutes.

\section{About the Author}

Dr. Fall is a postdoctoral student in the Department of Virology at the Pasteur Institute of Dakar. His research interest is the molecular characterization of noninfluenza respiratory viruses (mainly enterovirus, rhinoviruses, and respiratory syncytial virus) in pediatric populations in Senegal.

\section{References}

1. Kubi JA, Mutocheluh M, Bonney JHK, Ampofo WK, Odoom JK. Molecular detection of enterovirus D68 among children with acute respiratory tract infection in Ghana. Afr J Lab Med. 2019;8:732. https://doi.org/10.4102/ ajlm.v8i1.732

2. Fall A, Ndiaye N, Jallow MM, Barry MA, Touré CSB, Kebe O, et al. Enterovirus D68 subclade B3 circulation in Senegal, 2016: detection from influenza-like illness and acute flaccid paralysis surveillance. Sci Rep. 2019;9:13881. https:// doi.org/10.1038/s41598-019-50470-z 
3. Fall A, Jallow MM, Kebe O, Kiori DE, Sy S, Goudiaby D, et al. Low circulation of subclade A1 enterovirus D68 strains in Senegal during 2014 North America outbreak. Emerg Infect Dis. 2019;25:1404-7. https://doi.org/10.3201/eid2507.181441

4. Dyda A, Stelzer-Braid S, Adam D, Chughtai AA, MacIntyre $\mathrm{CR}$. The association between acute flaccid myelitis (AFM) and enterovirus D68 (EV-D68) - what is the evidence for causation? Euro Surveill. 2018;23. https:/ / doi.org/10.2807/1560-7917.ES.2018.23.3.17-00310

5. Centers for Disease Control and Prevention. Revision to the standardized surveillance and case definition for acute flaccid myelitis. 2018 [cited 2020 May 25]. https:/ / wwwn. cdc.gov/nndss/conditions/acute-flaccid-myelitis/ case-definition/2018

6. Sejvar JJ, Lopez AS, Cortese MM, Leshem E, Pastula DM, Miller L, et al. Acute flaccid myelitis in the United States, August-December 2014: results of nationwide surveillance. Clin Infect Dis. 2016;63:737-45. https:/ / doi.org/10.1093/ cid/ciw372

7. Messacar K, Asturias EJ, Hixon AM, Van Leer-Buter C, Niesters HGM, Tyler KL, et al. Enterovirus D68 and acute flaccid myelitis - evaluating the evidence for causality. Lancet Infect Dis. 2018;18:e239-47. https:/ / doi.org/10.1016/ S1473-3099(18)30094-X

8. Knoester M, Helfferich J, Poelman R, Van Leer-Buter C, Brouwer OF, Niesters HGM; 2016 EV-D68 AFM Working Group. Twenty-nine cases of enterovirus-D68-associated acute flaccid myelitis in Europe 2016: a case series and epidemiologic overview. Pediatr Infect Dis J. 2019;38:16-21. https://doi.org/10.1097/INF.0000000000002188

9. Ruggieri V, Paz MI, Peretti MG, Rugilo C, Bologna R, Freire C, et al. Enterovirus D68 infection in a cluster of children with acute flaccid myelitis, Buenos Aires, Argentina, 2016. Eur J Paediatr Neurol. 2017;21:884-90. https:/ / doi.org/ 10.1016/j.ejpn.2017.07.008

10. $\mathrm{Hu}$ YL, Chang LY. Current status of enterovirus D68 worldwide and in Taiwan. Pediatr Neonatol. 2019. https://doi.org/10.1016/j.pedneo.2019.09.007

11. Chen IJ, Hu SC, Hung KL, Lo CW. Acute flaccid myelitis associated with enterovirus D68 infection: a case report. Medicine. 2018;97:e11831. https://doi.org/10.1097/ MD.0000000000011831

12. Wang G, Zhuge J, Huang W, Nolan SM, Gilrane VL, Yin C, et al. Enterovirus D68 subclade B3 strain circulating and causing an outbreak in the United States in 2016. Sci Rep. 2017;7:1242. https:// doi.org/10.1038/ s41598-017-01349-4

13. Knoester M, Schölvinck EH, Poelman R, Smit S, Vermont CL, Niesters HG, et al. Upsurge of enterovirus D68, the Netherlands, 2016. Emerg Infect Dis. 2017;23:140-3. https://doi.org/10.3201/eid2301.161313

14. Carballo CM, Erro MG, Sordelli N, Vazquez G, Mistchenko AS, Cejas C, et al. Acute flaccid myelitis associated with enterovirus D68 in children, Argentina, 2016. Emerg Infect Dis. 2019;25:573-6. https:/ / doi.org/10.3201/ eid2503.170897

15. Pan American Health Organization/World Health Organization. Epidemiological alert: acute flaccid myelitis associated with enterovirus D68. Washington (DC): The Organizations; 2017 Nov 1 [cited 2020 May 28]. https://www.paho.org/hq/dmdocuments/2017/2017nov-01-epi-update-EV-D68.pdf

Address for correspondence: Ndongo Dia, Institut Pasteur Dakar, 36, Avenue Pasteur, B.P. 220, Dakar, Senegal; email: ndia@pasteur.sn

\section{EID Podcast: The Red Boy, the Black Cat}

Byron Breedlove, managing editor of Emerging Infectious Diseases, discusses the June 2019 EID cover artwork, a painting of Don Manuel Osorio Manrique de Zuniga, by Francisco de Goya y Lucientes.

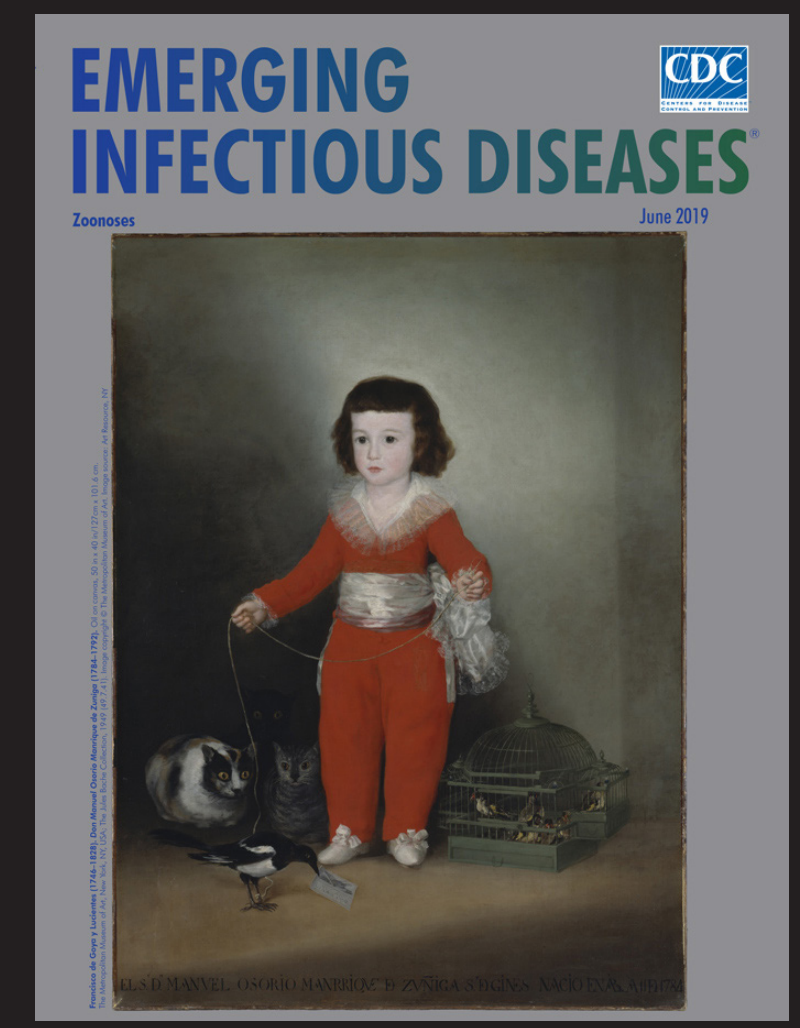

Visit our website to listen: https://go.usa.gov/xysv5

EMEREING INFECTIOUS DISEASES 